\title{
Controlled Assembly and Dispersion of Strain-Induced InGaAs/GaAs Nanotubes
}

\author{
Ik Su Chun and Xiuling Li
}

\begin{abstract}
Group III-V semiconductor nanotubes (SNTs) are formed when strained planar bilayers are released from the substrate. Compared to other nanotechnology building blocks, one of the main advantages of SNTs is the capability of precise positioning due to the top-down fabrication approach. In this letter, we demonstrate large-area assembly of ordered arrays of $\operatorname{In}_{x} \mathbf{G a}_{1-x} \mathrm{As} / \mathbf{G a A s}$ nanotubes and the dispersion of their freestanding form into solution and onto foreign substrates. In addition, we systematically investigate the crystal orientation dependence of rolling behavior using a wheel configuration, which serves as a guide for assembly homogeneity. Theoretical and experimental evaluations of tube diameters are also discussed.
\end{abstract}

Index Terms-Gallium compounds, indium compounds, nanotechnology, semiconductor materials.

\section{INTRODUCTION}

$\mathbf{T}$ HE MOST widely studied type of nanotubes is perhaps the carbon nanotubes (CNTs), first introduced by Iijima et al. in 1991 [1]. CNTs are formed by bottom-up methods such as chemical vapor deposition. The material architecture has proven to be of great interest for applications in nanoelectronic devices, solar cells, and chemical and biological sensing, etc.

Group IV and III-V compound semiconductor nanotubes (SNTs), a new paradigm, are formed by an entirely different process, a top-down approach. They were first fabricated by Prinz et al. in 2000, using strain-induced self-rolling of semiconductor bilayers grown by molecular beam epitaxy [2]. The rolling mechanism of SNTs is illustrated in Fig. 1, using a bilayer consisting of thin InAs and GaAs films and an AlAs sacrificial layer grown on a GaAs substrate. InAs has a larger lattice constant than the substrate [Fig. 1(a)], and thus, is compressively strained [Fig. 1(b)], as long as the critical thickness is not exceeded. When it is released from the substrate by undercutting through selective etching of the AlAs layer [Fig. 1(c)], InAs has the tendency to relax and expand to its original lattice constant. It is, however, constrained by the top GaAs layer that resists the expansion to maintain its lattice constant. As a result, the moment of force $\mathbf{M}$ is generated within the bilayer to create a rolled-up geometry, where the outer wall accommodates the layer with larger lattice constant and the layer with smaller

Manuscript received December 6, 2007; revised January 6, 2008. The review of this paper was arranged by Associate Editor J. Rogers.

I. S. Chun is with the Department of Materials Science and Engineering, University of Illinois, Urbana, IL 61801 USA.

$\mathrm{X}$. Li is with the Department of Electrical and Computer Engineering, Department of Materials Science and Engineering, and the Beckman Institute for Advanced Science and Technology, University of Illinois, Urbana, IL 61801 USA (e-mail: xiuling@uiuc.edu).

Color versions of one or more of the figures in this paper are available online at http://ieeexplore.ieee.org.

Digital Object Identifier 10.1109/TNANO.2008.926372

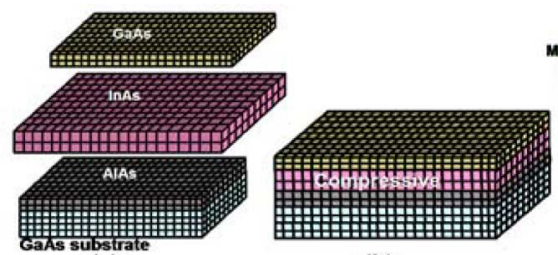

(a) (b)

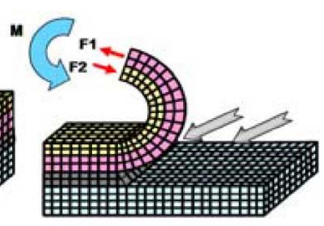

(c)
Fig. 1. Formation mechanism of strain-induced self-rolling of III-V semiconductor nanotube, illustrated with InAs/GaAs bilayer and AlAs sacrificial layer (adapted from [4]).

lattice constant resides in the inner wall. In general, as long as the two layers are relatively strained to each other, the rolling action takes place when they are detached from the substrate. SNTs formed this way were previously named as RUNTs for rolled-up nanotubes [3].

\section{EXPERIMENTAL}

In order to form SNTs using the strain-driven mechanism, the first step is to grow the planar strained bilayer structure epitaxially. Metalorganic chemical vapor deposition (MOCVD) was used for this study. ( 100 ) on-axis GaAs was the growth substrate. The structures include an $\mathrm{Al}_{x} \mathrm{Ga}_{1-x} \mathrm{As}(x>0.6)$ sacrificial layer with thickness varied from $50 \mathrm{~nm}$ to $2 \mu \mathrm{m}$, followed by a strained bilayer consisting of an $\operatorname{In}_{x} \mathrm{Ga}_{1-x} \mathrm{As}(x=0.2$ and 0.3 ) and a GaAs layer with nominal thickness of $6 \mathrm{~nm}$ each. The next step is to expose the sacrificial layer to etchant so that the bilayer can be released from the substrate. In the work presented here, photolithographic patterning was used to define the undercut mesa for bilayer detachment. The mask contained arrays of squares and rectangles with width and length ranging from 1 to $50 \mu \mathrm{m}$, and with varied orientations. Detailed patterning procedure was described previously [5]. SEM imaging was used to examine the topography, diameter of the formed tubes. Composition and thickness of the planar films were determined by energy dispersion spectroscopy (EDS) and scanning transmission electron microscopy (STEM), respectively.

\section{RESULTS AND DISCUSSION}

Based on the SNT formation mechanism, its diameter is determined by the bilayer strain and thickness. A macroscopic continuum mechanical model has been used to evaluate the tube diameter $D$ using the following equation [6]-[8]:

$$
D=\frac{\left.d\left[3(1+m)^{2}\right)+(1+m \cdot n)\left[m^{2}+(m \cdot n)^{-1}\right]\right]}{3 \varepsilon(1+m)^{2}}
$$




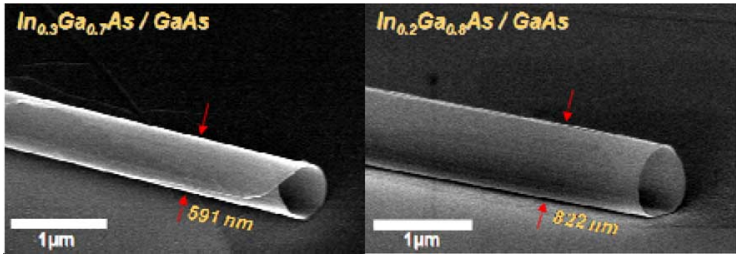

Fig. 2. Individual tube SEM images with diameter and structure labeled.

where $d$ is the total thickness $\left(d_{1}+d_{2}\right), m$ is the thickness ratio $\left(d_{1} / d_{2}\right), n$ is the Young's modulus ratio $\left(Y_{1} / Y_{2}\right)$, and $\varepsilon$ is the lattice mismatch $\left[\left(d_{2}-d_{1}\right) / d_{1}\right]$ between the two layers. Good agreement with experimentally measured radii as a function of thickness has been found, but in most cases, the experimental data are somewhat smaller than the theoretical values. The discrepancy in most cases can be corrected by reasonable assumptions of thickness deviation or additional strain [5], [7]-[11]. We have varied the composition in the $\operatorname{In}_{x} \mathrm{Ga}_{1-x} \mathrm{As} / \mathrm{GaAs}$ bilayer structure from $x=0.2$ to 0.3 . The diameters obtained from the rolled-up tubes (see Fig. 2) are smaller than the theoretical values by $\sim 14 \%$, corresponding to an additional strain of 0.003 . Surface effects, including tension and possible surface reconstruction, are possible sources of additional strain [12]-[14].

Taking advantage of the top-down formation process, we have fabricated large areas of highly ordered arrays of $\mathrm{In}_{0.3} \mathrm{Ga}_{0.7} \mathrm{As} / \mathrm{GaAs}$ nanotubes, as shown in Fig. 3. The tubes are well aligned to the lithographically defined rectangular mesa patterns, due to the crystal orientation dependence and geometry effect [5], [15]-[18]. The dimensions are $50 \mu \mathrm{m}$ in length and $\sim 590 \mathrm{~nm}$ in diameter, with an aspect ratio of $\sim 85$. The uniformity in the tube diameter measured along a tube and between different tubes is $9 \%$ in standard deviation. This is similar to a previous report [16] on $\mathrm{Ga}_{x} \operatorname{In}_{1-x} \mathrm{P}$ bilayer tubes that were $\sim 2 \mathrm{~mm}$ long and $1 \mu \mathrm{m}$ in average diameter. We attribute the diameter inhomogeneity to possible deviation of lithography patterns away from the crystal orientation that determines the rolling direction, as well as the variation in etch rate [15].

It is worth pointing out that these SNTs are completely released from the substrate where no chemical bond exists between the substrate and the tubes. This indicates that surface tension might be responsible for the adhesion that holds the SNTs in place. Such arrays of SNTs should be readily lift off by a soft-contact transfer process and print to other substrates [19].

The transferability of these SNTs can be simply demonstrated by removing them from the surface and dispersing into solution. Shown in Fig. 4 is a bundle of $\mathrm{In}_{0.2} \mathrm{Ga}_{0.8} \mathrm{As} / \mathrm{GaAs}$ SNTs freed from GaAs substrates by sonicating in methanol bath, then depositing onto a silicon substrate using a pipette. Most of the tubes survive this transfer process without breakage, maintaining the tubular structural integrity (Fig. 4, inset). A successful dispersion of functional freestanding tubes into the solution could enable applications as sensors in a test tube.

To ensure uniformity for large-area fabrication in diameter and orientation along an individual tube and from tube to tube, factors affecting the rolling behavior must be controlled carefully. To systematically investigate the orientation dependence

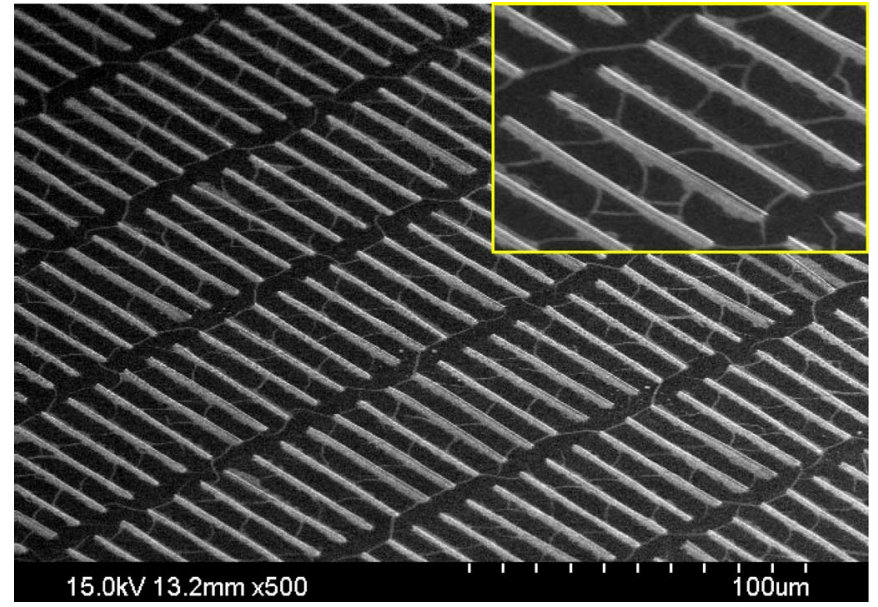

Fig. 3. Ensemble of $\operatorname{In}_{0.3} \mathrm{Ga}_{0.7} \mathrm{As} / \mathrm{GaAs}$ nanotubes that are $50 \mu \mathrm{m}$ in length and $\sim 590 \mathrm{~nm}$ in diameter, grown by MOCVD epitaxy and fabricated by lithographic patterning and selective etching. Zoomed-in image is shown as an inset at the upper right corner.

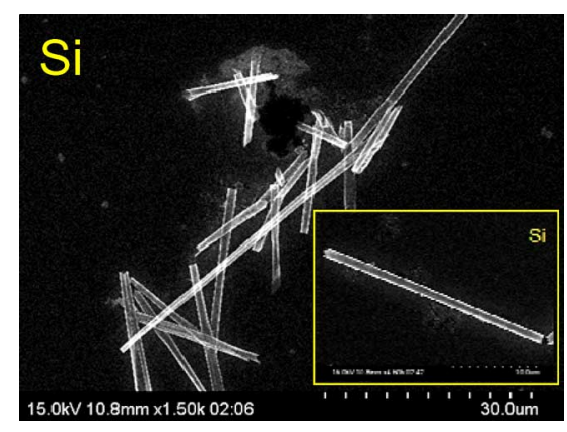

Fig. 4. SEM image of freestanding $\mathrm{In}_{0.2} \mathrm{Ga}_{0.8} \mathrm{As} / \mathrm{GaAs} \mathrm{SNT}$ deposited on a Si substrate. Lower right inset shows a single tube maintaining original tubular shape when the end is fractured.

of nanotube formation, we have used a wheel pattern. Shown in Fig. 5(a) is a lithographically patterned $\operatorname{In}_{0.3} \mathrm{Ga}_{0.7} \mathrm{As} / \mathrm{GaAs}$ film with the wheel configuration before undergoing etching to release the bilayer from substrate. The orientations of the eight anchored rectangular stripes are centrally symmetric along $\left\langle\begin{array}{lll}1 & 0 & 0\end{array}\right\rangle$ or $\left\langle\begin{array}{lll}1 & 1 & 0\end{array}\right\rangle$ direction. The dimension of each pad is $35 \mu \mathrm{m}$ long and $20 \mu \mathrm{m}$ wide, with 5- $\mu \mathrm{m}$-wide anchors.

The resulting topography of the pattern in Fig. 5(a) released from substrate is shown in Fig. 5(b). The center image shows all pads around the wheel while the zoomed-in images for each pad is laid out in outer periphery. It can be seen that tubes are formed from all pads along the diagonal lines ( $\left.\begin{array}{lll}1 & 0 & 0\end{array}\right\rangle$ direction). For the four pads oriented along the two axes $\left(\begin{array}{lll}\langle & 1 & 0\end{array}\right)$ directions $)$, rolling still occurs in the $\left\langle\begin{array}{lll}1 & 0 & 0\end{array}\right\rangle$ directions, which are along the corners of the free edges for these pads and thus "turn-over" triangular patterns are formed.

The persistence of rolling along the $\left\langle\begin{array}{lll}1 & 0 & 0\end{array}\right\rangle$ direction, independent of the stripe pattern orientation, apparently results from the anisotropy of stiffness in cubic crystals [4], [10], [20], [21]. It is evident that deviation from the $\left\langle\begin{array}{lll}1 & 0 & 0\end{array}\right\rangle$ rolling direction would lead to a twist of rolling, thus nonuniformity in diameter. 


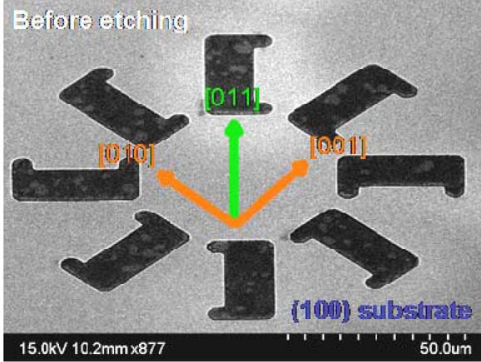

(a)

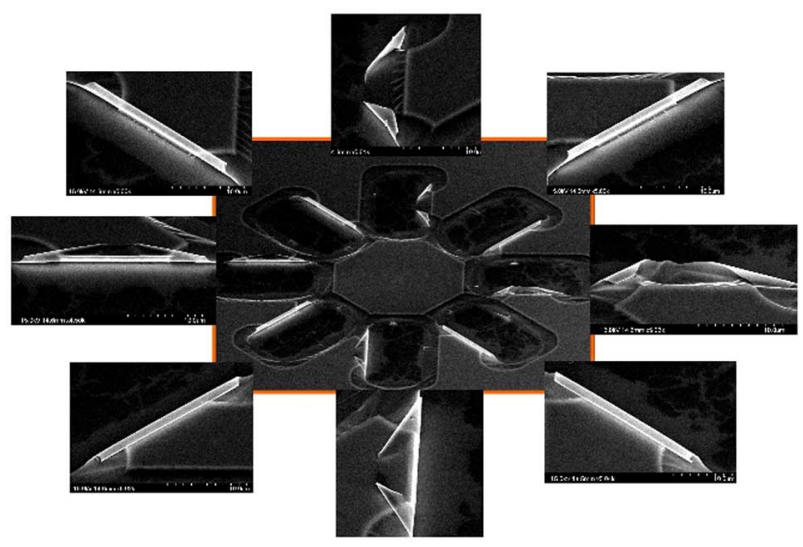

(b)

Fig. 5. (a) Before etching SEM image of lithographically patterned $\mathrm{In}_{0.3} \mathrm{Ga}_{0.7} \mathrm{As} / \mathrm{GaAs}$ film grown on $\left(\begin{array}{lll}1 & 0 & 0\end{array}\right) \mathrm{GaAs}$ substrate with a wheel of anchored rectangular stripe patterns. Crystal orientations are labeled. (b) After etching SEM images of the wheel pattern (center) with higher magnification images of each pad shown by the side.

On the other hand, the elastic anisotropy provides the basis for fabricating sophisticated 3-D architectures [17].

\section{CONCLUSION}

Strain-induced self-rolling semiconductor nanotubes and related structures represent a new class of building blocks of nanotechnology. The top-down fabrication approach allows largearea assembly with high precision, although uniformity could be challenging for smaller nanotubes. The dispersion of functional SNTs onto a solid surface of choice and into solution could lead to flexible sensing applications. With well-controlled formation patterns and sizes, along with the versatility of compound semiconductor in junction formation and bandgap tuning, SNTs hold the potential for yet another paradigm of nanotechnology.

\section{REFERENCES}

[1] S. Iijima, "Helical microtubules of graphitic carbon," Nature, vol. 354, pp. 56-58, 1991.

[2] V. Y. Prinz, V. A. Seleznev, A. K. Gutakovsky, A. V. Chehovskiy, V. V. Preobrazhenskii, M. A. Putyato, and T. A. Gavrilova, "Free-standing and overgrown InGaAs/GaAs nanotubes, nanohelices and their arrays," Physica E, vol. 6, pp. 828-831, 2000.
[3] C. Deneke, N. Y. Jin-Phillipp, I. Loa, and O. G. Schmidt, "Radial superlattices and single nanoreactors," Appl. Phys. Lett., vol. 84, pp. 4475-4477, 2004.

[4] V. Y. Prinz, "A new concept in fabricating building blocks for nanoelectronic and nanomechanic devices," Microelectron. Eng., vol. 69, pp. 466475, 2003.

[5] I. S. Chun, V. B. Verma, V. C. Elarde, S. K. Kim, J. M. Zuo, J. J. Coleman, and $\mathrm{X}$. Li, "InGaAs/GaAs 3D architecture formation by straininduced self-rolling with lithographically defined rectangular stripe arrays," J. Cryst. Growth, vol. 310, pp. 2353-2358, 2008.

[6] Y. C. Tsui and T. W. Clyne, "Analytical model for predicting residual stresses in progressively deposited coatings. Part 3: Further development and applications," Thin Solid Films, vol. 306, pp. 52-61, 1997.

[7] C. Deneke, C. Muller, N. Y. Jin-Phillipp, and O. G. Schmidt, "Diameter scalability of rolled-up $\operatorname{In}(\mathrm{Ga}) \mathrm{As} / \mathrm{GaAs}$ nanotubes," Semicond. Sci. Technol., vol. 17, pp. 1278-1281, 2002.

[8] P. O. Vaccaro, K. Kubota, and T. Aida, "Strain-driven self-positioning of micromachined structures," Appl. Phys. Lett., vol. 78, pp. 2852-2854, 2001.

[9] C. Giordano, M. T. Todaro, A. Salhi, L. Martiradonna, I. Viola, A. Passabi, L. Carbone, G. Gigli, A. Passaseo, and M. De Vittorio, "Nanocrystals cylindrical microcavities exploiting thin-walled InGaAs/GaAs microtubes," Microelectron. Eng., vol. 84, pp. 1408-1411, 2007.

[10] L. Zhang, S. V. Golod, E. Deckardt, V. Prinz, and D. Grutzmacher, "Freestanding Si/SiGe micro- and nano-objects," Physica E, vol. 23, pp. 280 284, 2004.

[11] O. Schumacher, S. Mendach, H. Welsch, A. Schramm, C. Heyn, and W. Hansen, "Lithographically defined metal-semiconductor-hybrid nanoscrolls," Appl. Phys. Lett., vol. 86, pp. 143109-1-143109-3, 2005.

[12] O. G. Schmidt and N. Y. Jin-Phillipp, "Free-standing SiGe-based nanopipelines on $\mathrm{Si}(0001)$ substrates," Appl. Phys. Lett., vol. 78, pp. 33103312, 2001.

[13] J. Zang and F. Liu, "Theory of bending of Si nanocantilevers induced by molecular adsorption: A modified Stoney formula for the calibration of nanomechanochemical sensors," Nanotechnology, vol. 18, pp. 4055011-405501-4, 2007

[14] Z. Ji, H. Minghuang, and L. Feng, "Mechanism for nanotube formation from self-bending nanofilms driven by atomic-scale surface-stress imbalance," Phys. Rev. Lett., vol. 98, pp. 146102-146400, 2007.

[15] H. Qin, N. Shaji, N. E. Merrill, H. S. Kim, R. C. Toonen, R. H. Blick, M. M. Roberts, D. E. Savage, M. G. Lagally, and G. Celler, "Formation of microtubes from strained SiGe/Si heterostructures," New J. Phys., vol. 7, no. 1, pp. 241-250, 2005.

[16] O. G. Schmidt, C. Deneke, S. Kiravittaya, R. Songmuang, H. Heidemeyer, Y. Nakamura, R. Zapf-Gottwick, C. Muller, and N. Y. Jin-Phillipp, "Selfassembled nanoholes, lateral quantum-dot molecules, and rolled-up nanotubes," IEEE J. Sel. Topics Quantum Electron., vol. 8, no. 5, pp. 10251034, Sep./Oct. 2002.

[17] V. Y. Prinz and S. V. Golod, "Elastic silicon-film-based nanoshells: formation, properties, and applications," J. Appl. Mech. Tech. Phys., vol. 47, pp. 867-878, 2006.

[18] O. G. Schmidt, C. Deneke, Y. M. Manz, and C. Muller, "Semiconductor tubes, rods and rings of nanometer and micrometer dimension," Phys. E: Low-Dimensional Syst. Nanostruct., vol. 13, pp. 969-974, Mar. 2002.

[19] Y. Sun, H.-S. Kim, E. Menard, S. Kim, I. Adesida, and J. A. Rogers, "Printed arrays of aligned GaAs wires for flexible transistors, diodes, and circuits on plastic substrates," Small, vol. 2, pp. 1330-1334, 2006.

[20] S. Mendach, O. Schmacher, C. Heyn, S. Schnull, H. Welsch, and W. Hansen, "Preparation of curved two-dimensional electron systems in InGaAs/GaAs-microtubes," Physica E, vol. 23, pp. 274-279, 2004.

[21] M. Huang, C. Boons, M. Roberts, D. E. Savage, M. G. Lagally, N. Shaji, H. Qin, R. Blick, J. A. Nairn, and F. Liu, "Nanomechanical architecture of strained bilayer thin films: From design principles to experimental fabrication," Adv. Mater., vol. 17, pp. 2860-2864, 2005.

Authors' photographs and biographies not available at the time of publication. 